\title{
Thermal dilepton rates and electrical conductivity of QGP from the lattice
}

\author{
Heng-Tong Ding \\ Key Laboratory of Quark \& Lepton Physics (MOE) and Institute of Particle Physics, Central \\ China Normal University, Wuhan 430079, China \\ E-mail: hengtong.ding@mail.ccnu.edu.cn
}

\section{Olaf Kaczmarek}

Fakultät für Physik, Universität Bielefeld, 33615 Bielefeld, Germany

E-mail: okacz@physik.uni-bielefeld.de

Florian Meyer*

Fakultät für Physik, Universität Bielefeld, 33615 Bielefeld, Germany

E-mail: fmeyer@physik.uni-bielefeld.de

\begin{abstract}
We present new results on the systematics of the reconstruction of mesonic spectral functions for three temperatures $1.1 T_{c}, 1.2 T_{c}$ and $1.4 T_{c}$ in quenched QCD. Using continuum extrapolated data, we extend our former analysis which used a well motivated Ansatz. Starting from this, two different systematics with respect to the shape of the Ansatz in the low frequency region are investigated. As a result, the covariance matrix turns out to measurably improve the fit. Furthermore, a second systematic verifies that a spectral function without a distinct transport peak describes our data as well. Thermal moments, extracted from the data, were found to play an important role in stabilizing the fits earlier. We find that also in the new systematics, the thermal moments, used to constrain the fit, are sensitive to the low frequency region as well.
\end{abstract}

The 33rd International Symposium on Lattice Field Theory

14 -18 July 2015

Kobe International Conference Center, Kobe, Japan*

${ }^{*}$ Speaker. 


\section{Introduction}

Ongoing Heavy Ion Collision experiments conducted at facilities like RHIC and LHC provide new output about the nature of elementary particles and their interactions. Direct photons and dileptons $\left(e^{+} e^{-}, \mu^{+} \mu^{-}\right)$are especially good probes of the QGP, as they are produced in every stage of its evolution and their coupling to other QGP constituents is small [1,2]; once they are produced, they leave the interaction region largely unmodified. The latest experiments PHENIX and STAR provide indications of thermal enhancements of dilepton spectra in the small to medium invariant mass region [3,4], which is the scale at which modifications by the surrounding thermal medium take place. On the other hand, the spectral function in the vector channel at finite temperature provides theoretical information on the thermal dilepton rates accessible in those experiments [5], which renders it a worthwhile object to study from theory. Especially the small frequency region of the spectral function contains information on important dynamical quantities like the charge diffusion constant and the electrical conductivity of the plasma [6]. Because this regime is also inherently non-perturbative, the use of lattice QCD data is implied. The following work is a systematic extension of our former investigations on the light vector spectral function at finite temperature [7], and discusses structural changes in the Ansatz and what information we can gain from these.

A well accessible quantity on the lattice is the correlation function in a given mesonic channel. It inhibits dynamical properties of the QGP state when investigated at finite temperature. As such, the light vector correlator is related to the electrical conductivity $\sigma$ of the QGP, the dilepton rate $\frac{\mathrm{d} W}{\mathrm{~d} \omega \mathrm{d}^{3} p}$ and the photon rate $\frac{\mathrm{d} R}{\mathrm{~d}^{3} p}$ as measured in heavy ion collision experiments, via its spectral function $\rho_{V}$ [8]. While in general spectral functions relate to correlators through an integral equation,

$$
G(\tau, \vec{p})=\int_{0}^{\infty} \frac{\mathrm{d} \omega}{2 \pi} \rho(\omega, \vec{p}, T) K(\omega, \tau, T) \quad \text { with } \quad K(\omega, \tau, T)=\frac{\cosh \left(\omega\left(\tau-\frac{1}{2 T}\right)\right)}{\sinh \left(\frac{\omega}{2 T}\right)}
$$

transport coefficients are related to the spectral functions via Kubo formulas. Examples of these are the shear and bulk viscosity obtained from energy momentum tensor correlation functions, the heavy quark momentum diffusion coefficient from color electric correlators [9], and the electrical conductivity, related to the light vector spectral function [7]. These relations imply that once the spectral function of the vector channel is extracted from QCD, important insights into nonperturbative phenomena of heavy ion collisions and the QGP can be gained.

In order to determine the spectral function, however, the Fredholm Type-I equation (1.1) has to be inverted, which is often referred to as an "ill posed" problem [6]. In our case it is a discrete problem, as we can access the value of the correlation function only at a finite number of points in $\tau T$. The basic idea is that the numerical (temporal) correlator data contains $\mathscr{O}(10)$ points, while a solution should be much more fine grained, ideally even continuous. This means there is more information desired on the r.h.s. than is actually provided on the l.h.s. Any approach to solving an ill posed problem, i.e. regain uniqueness and stability, must add information in order to "regularize" the problem and thus render it at least "better posed", with an important approach being the 


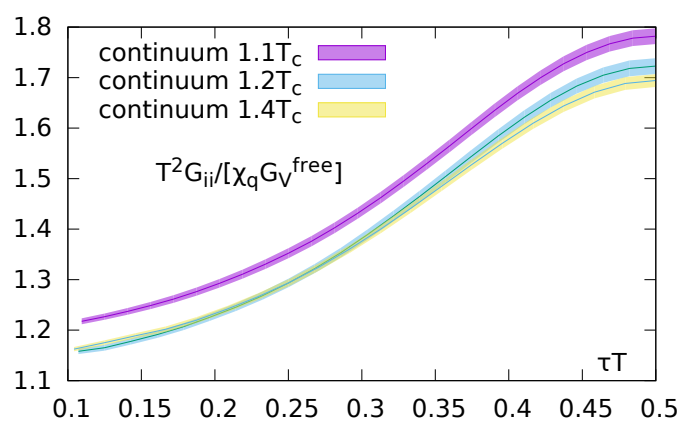

Figure 1: The continuum extrapolations for all three temperatures. The difference between the data at $T=1.1 T_{c}$ and the other two datasets is attributed to originate from a difference in the corresponding susceptibilities.

Bayesian methods. In the present work we choose the necessary additional information to enter the procedure in the form of a phenomenologically inspired Ansatz, which is fitted to continuum extrapolated lattice QCD correlation functions.

\section{Lattice setup}

The renormalized isovector correlation function is constructed as

$$
J_{h}=Z_{V} \bar{\psi}(x) \gamma_{h} \psi(x) \rightarrow G_{H}(\tau, \vec{x})=\left\langle J_{h}(\tau, \vec{x}) J_{h}^{\dagger}(0, \overrightarrow{0})\right\rangle \rightarrow G_{H}(\tau, \vec{p})=\sum_{\vec{x}} G_{H}(\tau, \vec{x}) e^{i \vec{p} \vec{x}},
$$

where $Z_{V}$ is the appropriate renormalization constant, non-perturbatively determined in [10], and $H=h h=00, i i$ is a specific component of the vector correlation function. The point to point correlators are projected to definite momentum $\vec{p}$ by summing over all spatial coordinates, In this study we constrain ourselves to the case $\vec{p}=0$. Splitting the correlation function (2.1) into spatially and temporally polarized components, defining for $H=V$ in Euclidean metric $G_{V}=G_{i i}+G_{00}$, we form a ratio of correlation functions

$$
R_{i i}=\frac{T^{2}}{\chi_{q}} \frac{G_{i i}(\tau T)}{G_{V}^{\text {free } l a t}(\tau T)}, \quad \text { with } \quad G_{i i} \equiv \sum_{i} G_{i i}, \quad \chi_{q}=-G_{00} / T,
$$

where $G_{i i}$ is normalized by both the free, massless correlator on the lattice [11], and the quark number susceptibility $\chi_{q} / T^{2}$. Lattice calculations have been performed in the quenched approximation using the standard Wilson gauge action and the non-perturbatively improved Wilson-Clover action [12] for the valence quarks. The hopping parameters $\kappa$ are tuned such that the valence quark masses are small, corresponding to $m_{\overline{M S}}(\mu=2 \mathrm{GeV}) \sim \mathscr{O}(10 \mathrm{MeV})$ in the $\overline{M S}$ scheme. For each temperature, three increasingly finer lattices with constant temperature are considered to allow for linear extrapolations in $a^{2}$ to the continuum, see [7, 13] for the details of the continuum extrapolation procedure. However, we stress here that an estimate of the continuum covariance matrix of the correlation function is available for all temperatures. A comparison of the continuum ratios for all three temperatures is shown in Fig. 1. Because the difference between $T=1.1 T_{c}$ and $T>1.1 T_{c}$ is attributed mostly to a difference in the quark number susceptibilities, we expect the underlying spectral functions also to be very similar for all three temperatures. 


\section{Fitting to the data}

In order to extract the vector spectral function via (1.1) we employ an Ansatz for its spatial part:

$$
\begin{aligned}
\rho_{\mathrm{ans}}(\omega, T) & =\chi_{q} c_{\mathrm{BW}} \frac{\omega \Gamma}{\omega^{2}+(\Gamma / 2)^{2}}+\frac{3}{2 \pi}(1+k) \omega^{2} \tanh \left(\frac{\omega}{4 T}\right) \\
& \equiv \rho_{\mathrm{BW}}(\omega, T)+(1+k) \rho_{V}^{\text {free }}(\omega, T) .
\end{aligned}
$$

It consists of two constituents: a Breit-Wigner peak, governing the behavior in the low $\omega$ region, and a modified version of the free, massless continuum spectral function. The modification parameter in the latter case fulfills $k=\alpha_{s} / \pi$ at leading order perturbation theory [13]. This Ansatz is inspired by the known relations for massless continuum spectral functions in the non-interacting case [14], especially

$$
\rho_{i i}^{\text {free }}(\omega, T)=2 \pi T^{2} \omega \delta(\omega)+\frac{3}{2 \pi} \omega^{2} \tanh \left(\frac{\omega}{4 T}\right) .
$$

The $\delta$-function in the spatial part is expected to be smeared out upon the onset of interactions [15]. Following the analysis in [13], motivated by arguments from kinetic theory, the $\delta$-function is modeled to become a Breit-Wigner peak $\rho_{B W}$ under this melting effect. The fit itself is performed by properly rescaling $\rho_{\text {ans }}$ with the free correlator and the quark number susceptibility, and especially by taking into account all statistical correlations among the data points by utilizing the corresponding covariance matrix of the continuum data. However, the information about the small $\omega$ region is most distinct in the large $\tau T$ region of the correlator [16], i.e. around its midpoint. We thus extract the second thermal moment from the continuum data, which is sensitive to this frequency region, and account for it in the fit procedure as an additional constraint, see [13].

\section{Results}

The fits with Ansatz $\rho_{\text {ans }}$ have been done in [7]. They work well for all three temperatures with $\chi^{2} /$ dof $\sim 1.1$, yielding estimates of the spectral function and thus electrical conductivities and dileptonrates. The spectral function at $T=1.1 T_{c}$ is shown here exemplarily in Fig. 3. However, the choice of the Ansatz is a central systematic in this procedure. In the next section we thus complement our earlier analysis by developing two structural changes in our Ansatz, and discuss what conclusion can be drawn from the respective modified Ansatz and its fit to the continuum extrapolated correlator data.

\subsection{Squeezing the Breit Wigner peak}

First of all, instead of using a Breit-Wigner peak for the low frequency part of the spectral function, we change it to be a real $\delta$ function with variable height,

$$
\rho_{\delta}(\omega)=a \chi_{q} \omega \delta(\omega)+(1+k) \rho_{V}^{\text {free }}(\omega) .
$$

Up to the parameter $k$ this is essentially the free case, see (3.1). The free case should yield an infinite conductivity, thus the above Ansatz $\rho_{\delta}$ should be incompatible with our interacting continuum 

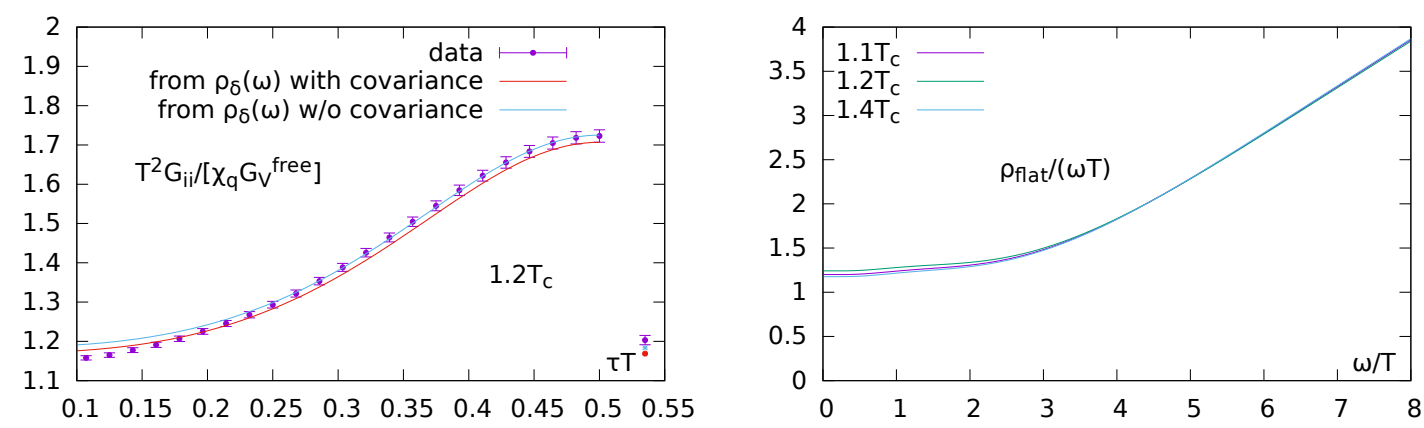

Figure 2: Left: Fit of a real delta peak in the low frequency region. The points at $\tau T=0.535$ are the thermal moment and its result, respectively. Note how the thermal moment is described much worse than any other data point. Right: The spectral function resulting from the fit of the (coarse) model $\rho_{\text {flat }}$ for all temperatures.

data. Performing the fit with Ansatz $\rho_{\delta}$ we find that the procedure yields values of $\chi^{2} / \operatorname{dof} \sim 1.5$ for the two lower temperatures, and $\chi^{2} /$ dof $\sim 2.5$, for $1.4 T_{c}$. Looking at the resulting correlators, shown examplarily in Fig. 2 (left) for $1.2 T_{c}$, we see that the curve obtained by fitting with the covariance matrix included really underestimates the correlator data points systematically by an amount of one standard deviation or more. Specifically, the fitted thermal moment, shown at $\tau T=0.535$ in the plot, drastically deviates from the data. We conclude that the Ansatz does not describe the data sufficiently and also place an emphasis on the importance of accurately determined thermal moments for the analysis. However, when we perform the fit without the covariance matrix in the minimizing $\chi^{2}$ term, we end up with a function that reconstructs the data points very well, even shows the usual small $\chi^{2} /$ dof $\sim \mathscr{O}(0.1)$, which is typical for missing correlations. The second thermal moment is still a bit off, however, albeit it describes the data point much better compared to the fit with the covariance matrix included. This again underlines the sensitivity of the thermal moments to the low frequency region. Apart from this, we can reverse the argument and see that the insufficient fit Ansatz $\rho_{\delta}$ fails to describe the data only if the information of the full covariance matrix is incorporated in the fit. In this sense we find that including covariances in the fit procedure measurably enhances our resolution of the spectral function in the low frequency region.

\subsection{Stretching the Breit Wigner peak}

Second, our Ansatz is motivated by kinetic theory and hydrodynamical arguments. On the other hand, in the strong coupling limit the vector spectral function can be obtained from the AdS/CFT correspondence. The resulting spectral function usually has no peak structure in the low frequency region [17], consisting for small $\omega$ of a flat, 'featureless' shape in $\rho / \omega$ and gradually turning into a typical large frequency behavior. A simple Ansatz roughly showing this behavior is given by

$$
\rho_{\text {flat }}(\omega)=a \chi_{q} \omega\left(1-\widetilde{\Theta}\left(\omega_{0}, \Delta_{0}\right)\right)+(1+k) \rho_{\text {free }}(\omega) \widetilde{\Theta}\left(\omega_{1}, \Delta_{1}\right) .
$$

The functions $\widetilde{\Theta}\left(\omega_{i}, \Delta_{i}\right)=\left(1+\exp \left(\frac{\omega_{i}^{2}-\omega^{2}}{\omega \Delta_{i}}\right)\right)^{-1}$ are smoothed Heaviside functions. This is of course a very rough model: there is some arbitrariness in the choice of $\omega_{i}$ and $\Delta_{i}$, and thus we do 

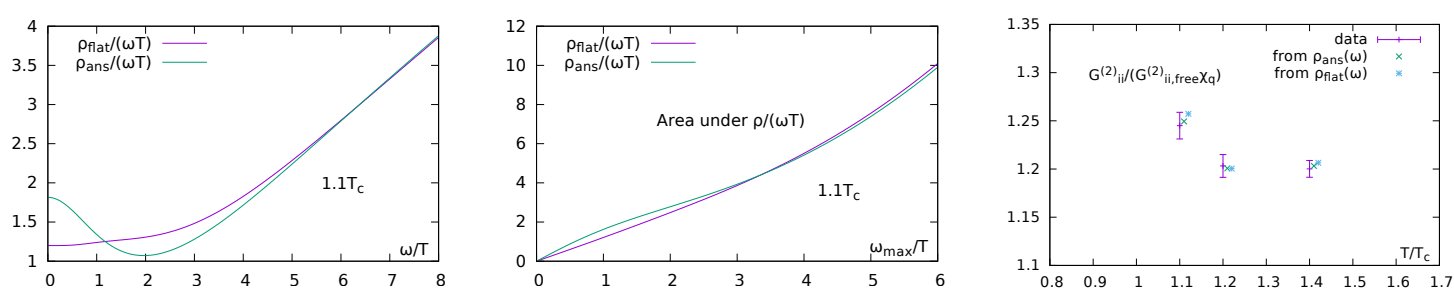

Figure 3: Left: The solutions of different Ansaetze compared for $T=1.1 T_{c}$. Note that the difference between $\rho_{\text {pert }}$ and $\rho_{\text {ans }}$ is most pronounced at $T=1.1 T_{c}$. Center: Integrating $\rho /(\omega T)$ up to $\omega_{\max }$, i.e. numerically computing its primitive function for $T=1.1 T_{c}$. Right: The thermal moments for all $T$ and their respective reconstructions from the fit, shown for all three models $\rho_{\text {ans }}, \rho_{\text {flat }}$ and $\rho_{\text {pert }}$.

not want to present definite results for dileptonrates or conductivities obtained from this Ansatz. But for our purpose of testing a non peaked, flat low frequency region, it will be appropriate. When fitting $\rho_{\text {flat }}$ to the data, we tune the cut positions $\omega_{i}$ and the smoothing parameters $\Delta_{i}$ in such a way that the result from the fit roughly describes the flat, featureless characteristic of the AdS/CFT solution. The fits work well for a range of cuts at $\omega_{i}$ and smoothing parameters $\Delta_{i}$, with $\chi^{2} /$ dof $\sim 1.1$ for $1.1 T_{c}, 1.4 T_{c}$ and $\chi^{2} /$ dof $\sim 0.5$ for $1.2 T_{c}$, see Fig. 2 (right) for the resulting spectral functions. The interpretation of this is first, that qualitatively this type of solution, being featureless in the low frequency region, fits our data just as well as a broad Breit-Wigner peak does. The resulting electrical conductivities, although somewhat dependent on $\omega_{i}$ and $\Delta_{i}$, are always located close to the lower bound of the ones obtained by fitting $\rho_{\text {ans }}$, see [7]. Comparing $\rho_{\text {ans }}$ and $\rho_{\text {flat }}$ in Fig. 3 (left), we see that, although the functions themselves have different shapes, the area under both is very similar in the low frequency region. Fig. 3 (center) shows the primitive integral of $\rho /(\omega T)$, which reveals that there is a range of frequencies, roughly $\omega / T \gtrsim 3$, for which the areas under the curves are the same. There is a sum rule found in perturbation theory [8], which states that the area under $\rho / \omega$ over the peak region is independent of the coupling, i.e. fixed for our purposes. This makes information about the exact shape of the spectral function for low frequencies difficult to obtain using euclidean data, because for small frequencies $K(\omega, \tau, T) \rightarrow 2 / \omega$, independent of $\tau$, and thus (1.1) is fulfilled for any spectral function whose low frequency region merely has the correct area. This effect we clearly also see in our fit procedure working on non-perturbative continuum data, and thus a flat shape like $\rho_{\text {flat }}$ naturally also leads to smaller electrical conductivities than e.g. $\rho_{\text {ans }}$. Because constraining the fits with the thermal moment is generally found to increase their quality, we also show the corresponding fit results in Fig. 3 (right). For $T=1.1 T_{c}$ and $T=1.4 T_{c}$ the fits work better for $\rho_{\text {ans }}$ than for $\rho_{\text {flat }}$, but the deviations of the latter are within error and, unlike for $\rho_{\delta}$, we cannot clearly differentiate the models this way without having a higher accuracy on the thermal moment.

\section{Conclusions}

We extent an earlier analysis of the light vector channel spectral function, which was done using quenched Wilson valence quarks extrapolated to the continuum at three temperatures. The continuum correlators show, within errors, no temperature dependence. Different systematics re- 
lated to the Ansatz are investigated. As a consequence, we find an essential improvement of the fit with respect to the low frequency region when performing the fit fully correlated, as opposed to neglecting the covariances of the data. Fitting an Ansatz inspired by the phenomenology of a strongly coupled QGP shows a comparable fit quality to the Ansatz motivated by a quasiparticle description, which implies that our procedure at this time does not resolve a difference between these two scenarios. This is also reflected by the fact that both spectral functions fulfill a sumrule valid in the low frequency region. The thermal moments, obtained from a seperate continuum extrapolation, are found to be sensitive to the low frequency region of the spectral function also in our systematic checks, and thus enhance the results from the fits. However, the fit to $\rho_{\text {flat }}$ shows the importance to have a high accuracy in the data, especially the thermal moments.

Acknowledgments: The results have been achieved using the PRACE Research Infrastructure resource JUGENE based at the Jülich Supercomputing Centre in Germany and the Bielefeld GPUcluster resources. This work has been partly supported by BMBF under grants 05P15PBCAA and the DAAD funding program 'Strategic Partnerships and Thematic Networks'.

\section{References}

[1] G. David, R. Rapp, and Z. Xu Phys.Rept. 462 (2008) 176-217, [nucl-ex/0611009].

[2] Y. Akiba PTEP 2015 (2015), no. 3 03A105.

[3] A. Adare et al. arXiv:1509.04667.

[4] STAR Collaboration Collaboration, A. et al. Phys. Rev. C 92 (Aug, 2015) 024912.

[5] D. Bernecker and H. B. Meyer Eur.Phys.J. A47 (2011) 148, [arXiv:1107.4388].

[6] H. B. Meyer Eur.Phys.J. A47 (2011) 86, [arXiv: 1104 .3708].

[7] H.-T. Ding, O. Kaczmarek, and F. Meyer arXiv:1412.5869.

[8] G. D. Moore and J.-M. Robert hep-ph/0607172.

[9] O. Kaczmarek Nucl.Phys. A931 (2014) 633-637, [arXiv:1409.3724].

[10] M. Luscher, S. Sint, R. Sommer, and H. Wittig Nucl.Phys. B491 (1997) 344-364, [hep-lat/9611015].

[11] S. Stickan, F. Karsch, E. Laermann, and P. Petreczky Nucl. Phys. Proc. Suppl. 129 (2004) 599-601, [hep-lat/0309191]. [,599(2003)].

[12] M. Luscher, S. Sint, R. Sommer, P. Weisz, and U. Wolff Nucl.Phys. B491 (1997) 323-343, [hep-lat/9609035].

[13] H.-T. Ding, A. Francis, O. Kaczmarek, F. Karsch, E. Laermann, et al. Phys.Rev. D83 (2011) 034504, [arXiv:1012.4963].

[14] G. Aarts and J. M. Martinez Resco Nucl.Phys. B726 (2005) 93-108, [hep-lat / 0507004 ].

[15] J. Hong and D. Teaney Phys.Rev. C82 (2010) 044908, [arXiv: 1003.0699 ].

[16] G. Aarts and J. M. Martinez Resco Nucl.Phys.Proc.Suppl. 119 (2003) 505-507, [hep-lat/0209033].

[17] D. Teaney Phys. Rev. D74 (2006) 045025, [hep-ph/ 0602044 ]. 